\section{BRAZIULIAN JOURNAL \\ OF MIEDICAL AND BIOLOGICAL RESFARCH}

www.bjournal.com.br
ISSN 0100-879X

Volume 42 (11) 993-1118 November 2009

BIOMEDICAL SCIENCES

AND

CLINICAL INVESTIGATION

Braz J Med Biol Res, November 2009, Volume 42(11) 1002-1014

Glutamate-N-methyl-D-aspartate receptor modulation and minocycline for the treatment of patients with schizophrenia: an update

C. Chaves, C.R. Marque, C. Trzesniak, J.P. Machado de Sousa, A.W. Zuardi, J.A.S. Crippa, S.M. Dursun and J.E. Hallak

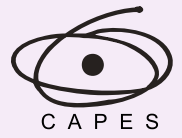




\title{
Glutamate-N-methyl-D-aspartate receptor modulation and minocycline for the treatment of patients with schizophrenia: an update
}

\author{
C. Chaves ${ }^{1,2}$, C.R. Marque ${ }^{1,2}$, C. Trzesniak ${ }^{1,2}$, J.P. Machado de Sousa ${ }^{1,2}$, \\ A.W. Zuardi ${ }^{1,2}$, J.A.S. Crippa ${ }^{1,2}$, S.M. Dursun ${ }^{2,3}$ and J.E. Hallak ${ }^{1,2}$ \\ ${ }^{1}$ Departamento de Neurociências e Ciências do Comportamento, Faculdade de Medicina de Ribeirão Preto, \\ Universidade de São Paulo, Ribeirão Preto, SP, Brasil \\ ${ }^{2}$ Instituto Nacional de Ciência e Tecnologia Translacional em Medicina (INCT-TM), \\ Faculdade de Medicina de Ribeirão Preto, Universidade de São Paulo, Ribeirão Preto, SP, Brasil \\ ${ }^{3}$ Department of Psychiatry, University of Alberta, Edmonton, Canada
}

\begin{abstract}
Growing consistent evidence indicates that hypofunction of N-methyl-D-aspartate (NMDA) transmission plays a pivotal role in the neuropathophysiology of schizophrenia. Hence, drugs which modulate NMDA neurotransmission are promising approaches to the treatment of schizophrenia. The aim of this article is to review clinical trials with novel compounds acting on the NMDA receptor (NMDA-R). This review also includes a discussion and translation of neuroscience into schizophrenia therapeutics. Although the precise mechanism of action of minocycline in the brain remains unclear, there is evidence that it blocks the neurotoxicity of NMDA antagonists and may exert a differential effect on NMDA signaling pathways. We, therefore, hypothesize that the effects of minocycline on the brain may be partially modulated by the NMDA-R or related mechanisms. Thus, we have included a review of minocycline neuroscience. The search was performed in the PubMed, Web of Science, SciELO, and Lilacs databases. The results of glycine and D-cycloserine trials were conflicting regarding effectiveness on the negative and cognitive symptoms of schizophrenia. D-serine and D-alanine showed a potential effect on negative symptoms and on cognitive deficits. Sarcosine data indicated a considerable improvement as adjunctive therapy. Finally, minocycline add-on treatment appears to be effective on a broad range of psychopathology in patients with schizophrenia. The differential modulation of NMDA-R neurosystems, in particular synaptic versus extrasynaptic NMDA-R activation and specific subtypes of NMDA-R, may be the key mediators of neurogenesis and neuroprotection. Thus, psychotropics modulating NMDA-R neurotransmission may represent future monotherapy or add-on treatment strategies in the treatment of schizophrenia.
\end{abstract}

Key words: Schizophrenia; NMDA agonist; Glycine; Glutamate; Clinical trial; Minocycline

\section{Introduction}

Schizophrenia is a disabling illness that affects approximately $1 \%$ of the world population (1) and is characterized by positive, negative and cognitive symptoms. This psychiatric disorder encompasses symptoms such as delusions, hallucinations, disorganized thinking, apathy, and deterioration of social role functioning (1). In addition, patients with schizophrenia experience severe suffering and close to $10 \%$ of them commit suicide (1). The typical onset of this disease is in young adults, and its course is often chronic (1).
Disruption of the dopaminergic system is implicated in the pathophysiology of schizophrenia. Conventional dopamine receptor-blocking antipsychotics are effective for positive symptoms, but they have limited effects on negative and cognitive symptoms (2). These symptoms possibly involve other neurotransmitter systems besides dopamine.

Emerging data indicate that hypofunction of glutamatergic N-methyl-D-aspartate receptor (NMDA-R) transmission plays a central role in the pathophysiology of schizophrenia $(3,4)$.

Correspondence: J.E. Hallak, Departamento de Neurociências e Ciências do Comportamento, HC, FMRP, USP, Av. Bandeirantes, 3900, 14048-900 Ribeirão Preto, SP, Brasil. E-mail: jhallak@fmrp.usp.br

Received March 14, 2009. Accepted September 15, 2009. Available online October 26, 2009. 
Hypothesis of NMDA-R hypofunction in schizophrenia

Emerging data indicate a direct involvement of the glutamatergic system in the pathophysiology of schizophrenia core symptoms. Complementary to the dopamine hypothesis, the hypofunction of the ionotropic glutamate NMDA-R has been proposed as a model of schizophrenia in humans (4). Thus far, there have been many clinical trials with novel compounds acting on the NMDA-R.

The glutamatergic hypothesis of schizophrenia postulates that the dysfunction of neurotransmission mediated by the NMDA-R might represent a primary deficit in this disorder (4). The ability of phencyclidine, ketamine, and other NMDA-R antagonists to induce schizophrenia-like symptoms in healthy volunteers and to exacerbate psychosis in schizophrenic patients is the most compelling evidence of a relationship between NMDA-R function and schizophrenia $(4,5)$.

The NMDA-R antagonist-induced psychosis models are closer to certain clinical aspects of schizophrenia than the amphetamine/dopamine model, mainly by the generation of negative symptoms and cognitive deficits $(4,5)$. Thus, the potentiation of NMDA-R transmission might be a promising treatment approach to schizophrenia.

\section{Drugs that modulate NMDA-R neurotransmission}

Since glutamate and direct-acting NMDA-R agonists may be neurotoxic and are known to cause excitotoxicity, the strategy used in the last decade has relied on agonists at the strychnine-insensitive glycine receptor site of the NMDA-R complex (6). Therefore, experiments have been carried out on full agonists like glycine, D-serine and D-alanine, on the partial agonist D-cycloserine, and on sarcosine.

\section{NMDA-R full agonists}

Glycine is a small naturally occurring amino acid that functions as an endogenous co-agonist at the strychnineinsensitive glycine binding site, being a prerequisite for the activation of the NMDA ion channel (7). Glycine is not synthesized by glutamatergic neurons and a small amount is released from glycine neurons, so that the neighboring glial cells are the main source of the glycine available for the NMDA-R (8). The permeation of glycine into the brain barrier after peripheral administration is the lowest for any naturally occurring amino acid and high oral doses of glycine must be administered to achieve significant elevations in its central nervous system (CNS) levels (7).

$D$-serine is an endogenous co-transmitter enriched in corticolimbic regions and distributed in parallel with the NMDA-R $(9,10)$, acting as an endogenous full agonist at the NMDA-R-associated glycine site $(9,10)$. D-serine can be derived from $\mathrm{L}$-serine by serine racemase (SR) enzymatic conversion $(8,9)$. Although initial studies have suggested that this enzyme was localized in glial cells, a recent investigation using novel SR knockout mice as negative controls pointed out that SR was co-localized with neuron-specific nuclear proteins, with SR expression being observed in GABAergic and glutamatergic neurons, but not in glia (9). In addition, serum and cerebrospinal fluid levels of $D$ - and $\mathrm{L}$-serine and $\mathrm{D}$-serine ratio ( $\mathrm{D}$-serine/total serine) may be altered in schizophrenia, as demonstrated by reports of reduction of the $D$-serine ratio in the cerebrospinal fluid of drug-naive schizophrenic patients (3) and by decreased serum levels of D-serine in patients with schizophrenia (5). These data suggest that D-serine synthesis or metabolization may be altered in schizophrenia, highlighting a possible reduction of SR activity $(3,5)$. Moreover, D-serine has high affinity for the glycine site on NMDA-R and has the advantage of being more permeable than glycine through the blood-brain barrier, thus requiring a reduced amount per dose $(8,10)$.

D-alanine, as well as D-serine, is an endogenous Damino acid present in the human CNS (11) and is a selective and potent agonist at the NMDA-R glycine site (11).

\section{NMDA-R partial agonist}

$\mathrm{D}$-cycloserine, an analog of D-serine, is an antituberculous drug rarely used nowadays due to its adverse psychiatric effects including anxiety, psychosis and seizures at doses of 500-2000 mg (12). D-cycloserine freely crosses the blood-brain barrier and has good bioavailability to the brain (13). It is a partial agonist at the glycine recognition site at lower doses, with agonist properties over a narrow dose range; hence, it can exhibit antagonist activity at higher concentrations. Additionally, D-cycloserine has approximately $40-60 \%$ activity compared to glycine and the glycine modulatory site of the NMDA-R (13).

\section{Glycine transporter-1 inhibitor}

Sarcosine (or N-methylglycine), a nonessential amino acid, is an endogenous antagonist of glycine transporter-1 (glycine-T1). Glycine-T1 is located in the glial cells and its reuptake pump is the main route of inactivation of synaptic glycine. Therefore, the inhibition of glycine-T1 reuptake can increase glycine levels in the glutamatergic synapses and consequently augment NMDA-R transmission (8). Sarcosine is a natural occurring glycine-T1, which potentiates the action of glycine on the NMDA glycine site and can enhance NMDA neurotransmission (14).

Glycinergic agents and targets of NMDA-R modulation are summarized in Figure 1.

\section{NMDA-R augmentation}

Recent studies have supported the idea that increasing NMDA-R function may be an effective approach to the treatment of schizophrenia (15). Two recent reviews have been published about the use of glycinergic agents for this purpose $(15,16)$. Tuominem et al. (15) published a systematic review and meta-analysis that comprised eighteen short-term trials using glycine, D-serine or D-cycloserine to increase the antipsychotic action. These investigators also 
included ampakine CX516, an experimental drug that acts as an a-amino-3-hydroxy-5-methylisoxazole-4-propionic acid (AMPA) receptor agonist. Glycine and D-serine were successful in reducing the negative symptoms of schizophrenia and the magnitude of the effect was moderate. D-cycloserine had a less efficient action on negative symptoms. On the other hand, positive symptoms failed to respond to glycinergic medication and there were no significant effects of glycine or D-serine on cognitive functioning.

Shim et al. (16), in a review of the results of glycinergic agents in the treatment of schizophrenia, proposed a polypharmacy of glycine site potentiators in addition to antipsychotic medication to stop neurodegeneration in schizophrenia. In addition, they suggested that drugs acting on other sites of the NMDA-R could also be effective in the treatment of the disorder.

\section{Hypothesis of NMDA-R modulation by minocycline}

Minocycline is a second-generation tetracycline with a broad spectrum of antimicrobial activities and anti-inflammatory properties. There is growing evidence showing neuroprotective effects of minocycline in several animal and human models of neurological diseases, including Parkin- son's disease, amyotrophic lateral sclerosis, Huntington's disease, and ischemia (17).

Translational neuroscientific data from animal and human studies indicate that minocycline may be a potential treatment for schizophrenia. In the published literature, the first evidence indicating the antipsychotic efficacy of minocycline was observed in a patient with Huntington's disease, a case report from Canada (18). This clinical observation indicated the potential antipsychotic efficacy of this drug in schizophrenia. Zhang et al. (19) observed that pretreatment of mice with minocycline $(40 \mathrm{mg} / \mathrm{kg})$ significantly attenuated the level of dopamine in the striatum after repeated administration of methamphetamine. The same group (20) found that pretreatment with minocycline $(40 \mathrm{mg} / \mathrm{kg})$ reduced the increase of extracellular dopamine levels in the frontal cortex and striatum of mice after administration of the NMDA-R antagonist dizolcipine. Moreover, subsequent subchronic administration of minocycline considerably reduced the cognitive deficits induced by the NMDA-R antagonist phencyclidine in mice (21) and reversed the cognitive effects of the NMDA-R antagonist MK801 in rats (22).

The neuroprotective action of minocycline can occur indirectly by inhibition of microglial activation and prolif-

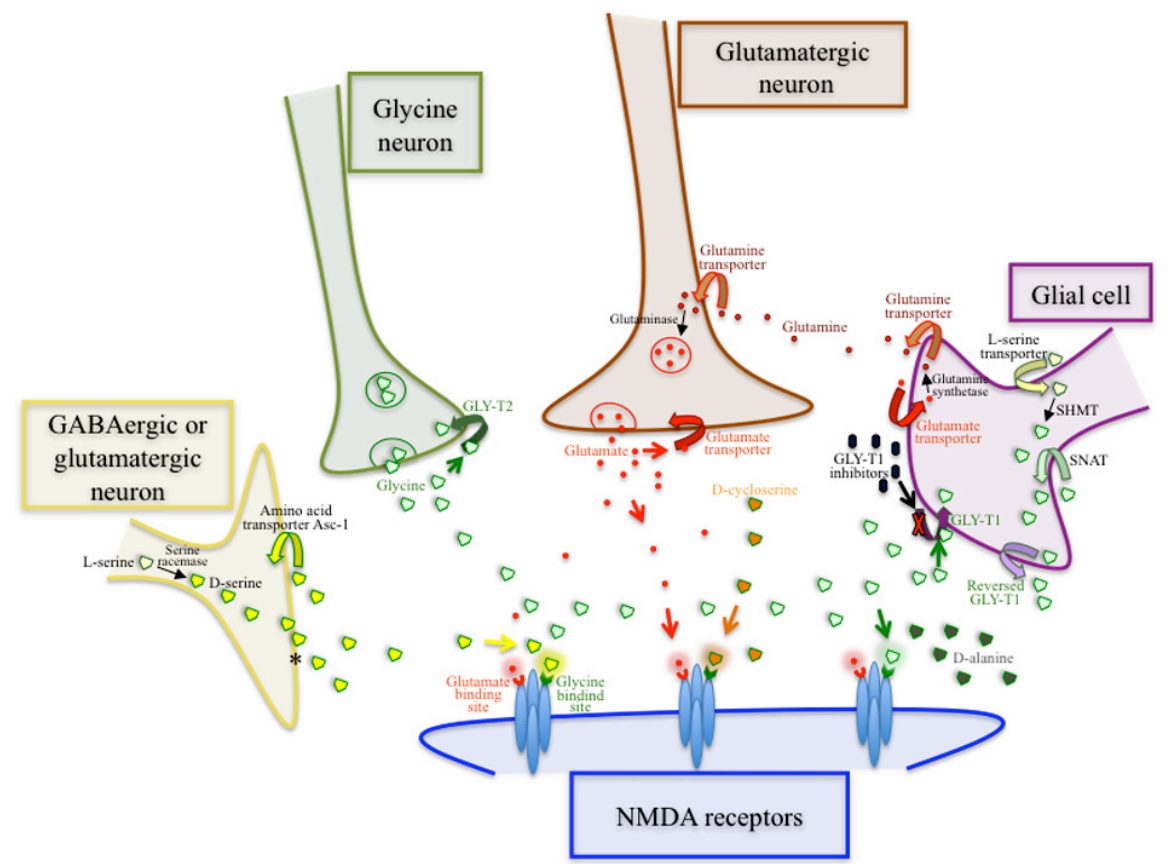

Figure 1. Allosteric modulation of NMDA-R transmission by glycinergic agents. NMDA = N-methyl-D-aspartate; GLY-T1 = type-1 glycine transporter; GLY-T2 = type-2 glycine transporter; SHMT = serine hydroxymethyl transferase; SNAT = specific neutral amino acid transporter. ${ }^{*}$ The pathway for D-serine release from neurons is unknown. Glycinergic agents (glycine, D-serine, D-alanine or D-cycloserine) are obligatory coagonists at the strychnine-insensitive glycine binding site of NMDA receptors. Small amounts of glycine are released from glycinergic neurons and are taken back up by a GLY-T2 located in these neurons. However, most of the glycine available in the synaptic cleft is released by glial cells through a reversed GLY-T1. Glycine can be taken up into glial cells by GLY-T1 or through a glial specific neutral amino acid transporter. Glycine can also be converted from L-serine in glial cells through SHMT action. It is noteworthy that glycine reuptake by GLY-T1 in glial cells limits the availability of glycine in the synaptic cleft. L-serine can also be converted to D-serine by serine racemase in GABAergic or glutamatergic neurons. D-serine availability in the synaptic cleft is limited through its reuptake by amino acid transporter Asc-1 located in these neurons. 
eration and nitric oxide synthase inhibition (17). There are also influences on caspase expression and cytochrome c release, with an anti-apoptotic property, among others (17). Although the precise mechanism of action of minocycline remains unclear, the latest studies conducted with this agent suggest effects related to the glutamatergic system, in addition to its action as an anti-apoptotic drug. In fact, minocycline reversed several NMDA-R antagonist effects in animal studies and showed good results in the treatment of patients with schizophrenia $(23,24)$. Thus, the hypothesis was raised that minocycline indirectly modulates NMDA-R transmission.

Furthermore, minocycline may exert a differential action on NMDA-R signaling pathways (described in the Discussion section). Consequently, the data about the three studies of minocycline in patients with schizophrenia were included in the present review.

The objective of this review is to identify and analyze clinical trials with NMDA-R transmission modulation and minocycline for the treatment of patients with schizophrenia.

\section{Methods}

The search was carried out in the PubMed, Web of Science, SciELO, and Lilacs databases using the key words schizophrenia, NMDA agonist, glycine, serine, glutamate, clinical trial, and minocycline, up to September 2008. It contemplated only clinical trials with NMDA-R transmission modulation in the treatment of schizophrenia, with no year or language limits. We also inspected the reference lists of the reviewed articles to identify additional trials. We excluded animal or in vitro studies, review or meta-analysis articles, and studies on healthy volunteers. The present review covered 36 clinical trials.

\section{Results}

There were 27 randomized placebo-controlled doubleblind studies $(2,6,7,10-12,14,23,25-42)$, 8 non-randomized trials (43-51), and 1 case report. Except for two trials $(42,49)$ with drug-free patients, all other studies involved adjuvant therapy for antipsychotic treatment. Clinical assessments were performed biweekly throughout most of the investigations.

The most used inclusion criteria were schizophrenia diagnosis according to the Diagnostic and Statistical Manual (DSM), primary deficit syndrome (using the Schedule for the Deficit Syndrome), minimum score of 30 on the Scale for the Assessment of Negative Symptoms (SANS), and use of a stable antipsychotic dose for more than three months. Exclusion criteria comprised comorbidity with substance abuse, major depression, serious clinical diseases, neurological diseases, and additional use of other psychotropic medications.

A combination of various scales was used for the evalu- ation of clinical efficacy: Positive and Negative Symptoms Scale (PANSS), Brief Psychiatric Research Scale (BPRS), SANS, Global Assessment Scale (GAS), and Clinical Global Impression (CGI). The PANSS and/or SANS were used in almost all of the studies. Eleven trials $(10,12,23,28,31,34$, $37,38,47,48,50)$ assessed specific cognitive tests.

To facilitate the presentation of the results, studies were divided into four groups: 1) glycine, 2) D-cycloserine, 3) Dalanine, D-serine and sarcosine, and 4) minocycline.

\section{Glycine}

Twelve articles were selected regarding the addition of glycine to the antipsychotic treatment as usual (Table 1), including 9 placebo-controlled double-blind trials (divided into 4 parallel and 5 crossover studies) $(2,7,25-31)$, and 3 open-label trials (43-45). The duration of the studies ranged from 6 to 28 weeks. The first studies $(25,43,44)$ used low doses, including 10, 15 and $30 \mathrm{~g} /$ day. However, the glycine dose needs to be higher to sufficiently cross the blood-brain barrier, and most of the trials subsequently administered higher doses (60 or $0.8 \mathrm{~g} \cdot \mathrm{kg}^{-1} \cdot \mathrm{day}^{-1}$ ).

For heuristic purposes, the results shown in Table 1 are analyzed in three distinct groups according to the type of antipsychotic used.

\section{Glycine added to conventional antipsychotics}

Eight trials evaluated the addition of glycine to typical antipsychotics $(25-27,29,31,43-45)$ and none of them observed any beneficial effect of glycine on positive symptoms. Rosse et al. (43) published a pilot study with 6 patients in which no significant results were obtained regarding the negative symptoms of schizophrenia. However, this study (43) was done with a low dose of glycine (10.8 g/day), which could explain the lack of effectiveness. The seven other investigations showed a beneficial effect on negative symptoms.

In 2007, Buchanan et al. (31) published the Cognitive and Negative Symptoms in Schizophrenia Trial (CONSIST), a randomized double-blind study with a duration of 16 weeks and with the participation of 4 sites in the United States and one site in Israel. A total of 157 patients were recruited and randomized to glycine, D-cycloserine and placebo groups. The study suggested that glycine is not an effective therapeutic option for treating negative symptoms. Nevertheless, post hoc analyses revealed that, among subjects receiving conventional antipsychotics, the 8 subjects randomly assigned to glycine had a greater decrease in the SANS total score compared to the 7 subjects randomly assigned to placebo. This trial also showed no significant difference in treatment response between patients with and without deficit syndrome. Nonetheless, tendencies to greater negative symptom reductions for both the glycine and D-cycloserine groups compared with placebo were observed in inpatients, but not in outpatients.

Moreover, the CONSIST (31) was the only study that 


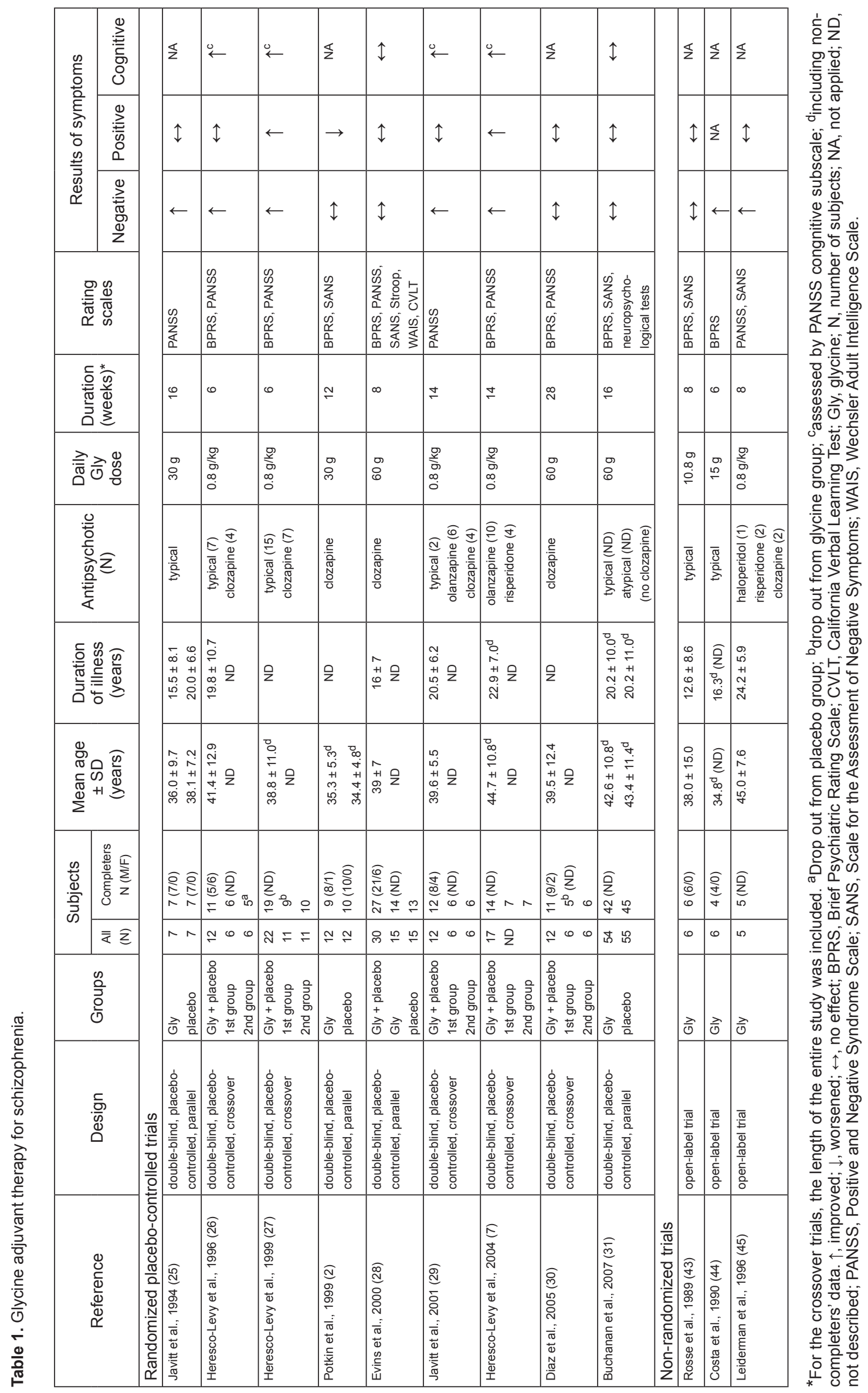


assessed specific cognitive tests, detecting no significant results compared to placebo. Three other trials $(26,27,29)$ evaluated the cognitive effects of glycine based on clinical ratings (PANSS cognitive subscale) rather than neuropsychological examination, showing a positive result in cognitive functioning. Nevertheless, it is noteworthy that these three investigations $(26,27,29)$ included a relatively small patient sample size and short-term glycine treatment.

\section{Glycine added to second-generation antipsychotics}

Four trials appraised the addition of glycine to secondgeneration antipsychotics $(7,29,31,45)$. A 6-week doubleblind crossover trial (7) registered a significant reduction of positive symptoms while the other three $(29,31,45)$ did not exhibit a significant change of positive symptoms.

Three studies $(7,29,45)$ provided evidence for a reduction of negative symptoms. However, these clinical trials were conducted on small samples over short periods of time, while the CONSIST (31) found no significant effect compared to placebo.

Once more, the CONSIST (31) was the only study that applied specific cognitive tests, obtaining no significant results compared to placebo. Two other short-term trials $(7,29)$ measured the cognitive effects of glycine based on clinical ratings (PANSS cognitive subscale) rather than on neuropsychological examination, pointing to a positive result in cognitive functioning.

\section{Glycine added to clozapine}

Seven trials $(2,26-30,45)$ evaluated the addition of glycine to clozapine. Five of them $(26,28-30,45)$ did not find a large change of positive symptoms with the adjuvant use of glycine in combination with clozapine. One study (2) found worsening of positive symptoms. Although one trial (27) reported a reduction of positive symptoms, the authors did not specify whether the results were related to the typical treatment or to the clozapine group.

Although four trials $(26,27,29,45)$ included patients on clozapine with a further decrease of negative symptoms, most of them $(26,27,45)$ did not distinguish between patients taking clozapine and patients taking other antipsychotics. Three other investigations $(2,28,30)$ showed no beneficial effects on negative symptoms.

Cognitive functioning was assessed in only four trials (2629). In one investigation (28), specific cognitive tests were applied and no effect on cognition was observed. The other three studies used the PANSS cognitive subscale, indicating beneficial results.

\section{D-cycloserine}

Fourteen articles were selected involving the addition of D-cycloserine to antipsychotic treatment as usual (Table 2), including 9 placebo-controlled double-blind trials $(6,12,31$ $37)$ and 5 non-randomized trials (46-50). These studies had a variable length of 4 to 24 weeks. Since D-cycloserine is a partial agonist, four studies (47-50) compared the effectiveness of $5,15,50$ and $250 \mathrm{mg} /$ day D-cycloserine in an attempt to obtain the optimal dosing. The initial results suggested that the dosage of $50 \mathrm{mg} /$ day yielded the best results, with worsening of symptoms at higher doses (100-250 mg/day) and lack of effect at lower doses (5-30 mg/day).

\section{D-cycloserine added to conventional antipsychotics}

Ten trials evaluated the addition of D-cycloserine to typical antipsychotics $(6,12,31-34,36,37,46,47)$. Seven of them $(6,12,31-34,47)$ did not find any significant change in positive symptoms and two (with higher dosages, namely 100 and 250 $\mathrm{mg} /$ day) reported worsening of positive symptoms $(36,46)$.

Beneficial effects on negative symptoms were obtained in four trials $(6,33,34,47)$, while four other studies $(12,31,32,37)$ did not find a significant change. Two studies $(36,46)$ with doses of 100 and $250 \mathrm{mg} /$ day showed worsening of negative symptoms.

In addition, one trial (47) demonstrated improvement of cognitive functioning at the dose of $50 \mathrm{mg} /$ day, while three other trials $(12,34,37)$ did not find significant changes in cognitive symptoms. The CONSIST (31) was the largest trial evaluating the addition of D-cycloserine to typical antipsychotics and showed no effect on positive, negative or cognitive symptoms.

\section{D-cycloserine added to second-generation antipsychotics}

Four trials evaluated the effect of $D$-cycloserine added to second-generation antipsychotics $(6,31,33,50)$ and none of them detected any significant change in positive symptoms. Three studies $(6,33,50)$ demonstrated a decrease of negative symptoms at the dose of $50 \mathrm{mg} / \mathrm{day}$. On the other hand, these trials were conducted on a small numbers of participants and the CONSIST (31) showed no significant effect of the increase of D-cycloserine to $50 \mathrm{mg} /$ day on negative symptoms. Only two trials $(31,50)$ assessed cognitive functioning and showed no significant effects.

\section{D-cycloserine added to clozapine}

Only three trials evaluated the addition of D-cycloserine to clozapine $(33,35,48)$, showing no significant effects on cognitive or positive symptoms. One trial (33) observed amelioration of negative symptoms, but did not distinguish the type of antipsychotics used and consisted of only 9 participants. The other two investigations showed worsening of negative symptoms when D-cycloserine was added to clozapine.

\section{D-alanine, D-serine and sarcosine}

Eight articles involving the addition of these drugs to the antipsychotic treatment were selected (Table 3).

\section{D-alanine}

There is only one trial involving the addition of D-alanine to habitual antipsychotic treatment (Table 3), with the addi- 


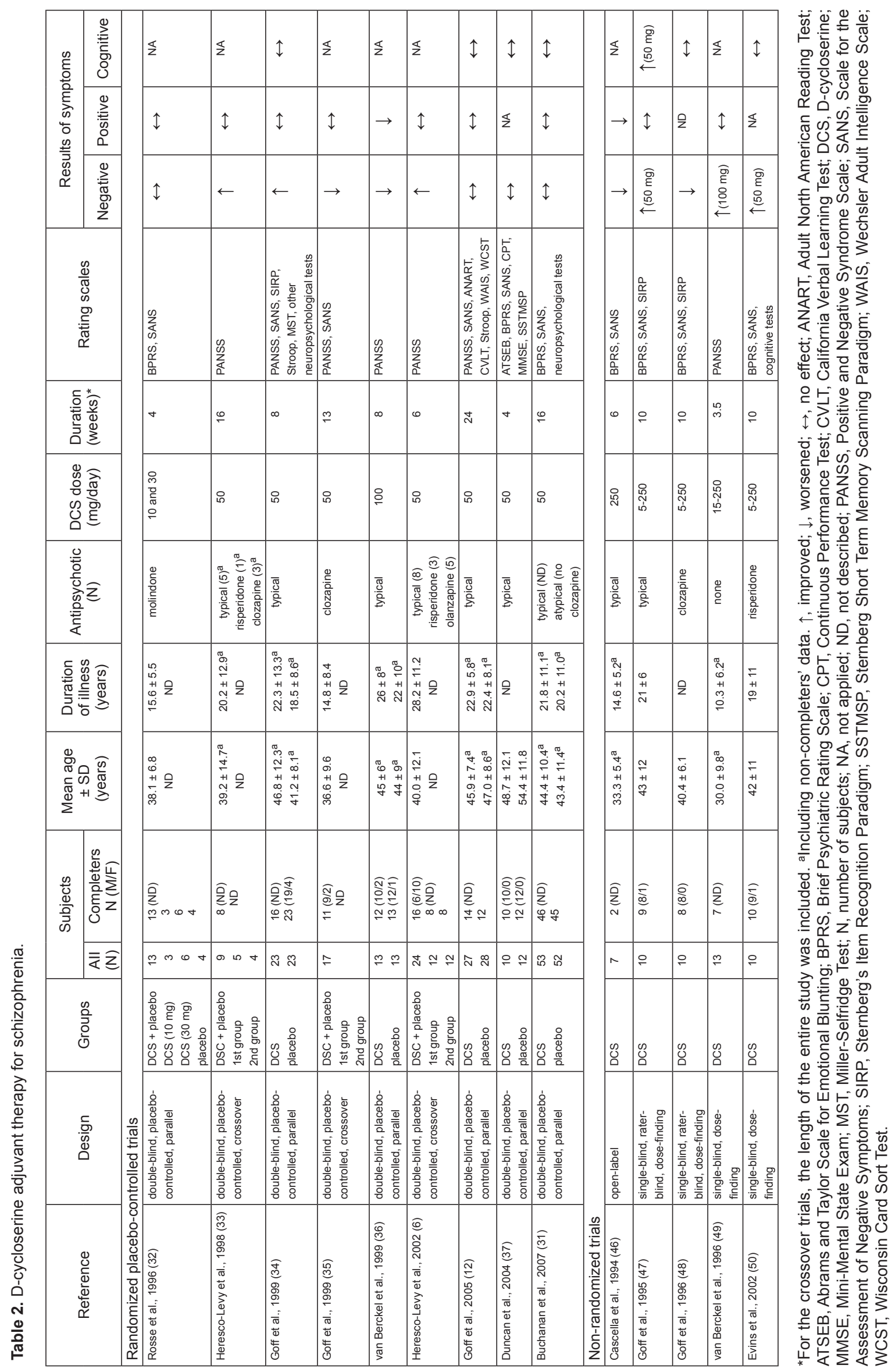




\begin{tabular}{|c|c|c|c|c|c|c|c|c|c|}
\hline \multirow{3}{*}{ 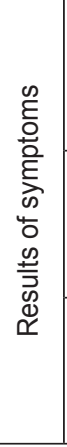 } & $\stackrel{\circ}{\leftarrow}$ & $\leftarrow$ & $\uparrow$ & $\stackrel{2}{\leftarrow}$ & $\stackrel{0}{\leftarrow}$ & 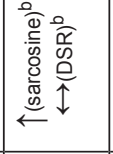 & 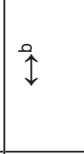 & $\frac{\pi}{z}$ & 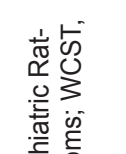 \\
\hline & $\leftarrow$ & $\leftarrow$ & $\uparrow$ & $\leftarrow$ & $\leftarrow$ & 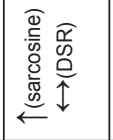 & $\uparrow$ & $\uparrow$ & 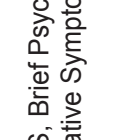 \\
\hline & $\leftarrow$ & $\leftarrow$ & $\uparrow$ & $\leftarrow$ & $\leftarrow$ & 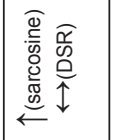 & $\uparrow$ & $\uparrow$ & 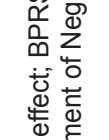 \\
\hline 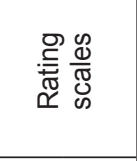 & $\begin{array}{l}0 \\
0 \\
\infty \\
\infty \\
\infty \\
\infty \\
0 \\
2 \\
0 \\
0\end{array}$ & 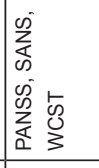 & 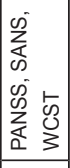 & 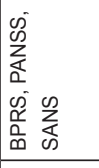 & 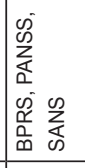 & 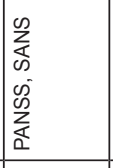 & \begin{tabular}{|l}
$\infty$ \\
0 \\
$\sum_{\alpha}^{\infty}$ \\
0 \\
\end{tabular} & $\begin{array}{l}0 \\
0 \\
\alpha \\
\infty \\
\infty \\
\infty \\
00 \\
0 \\
0 \\
0\end{array}$ & 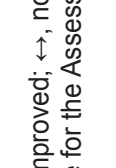 \\
\hline 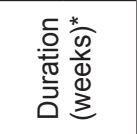 & 0 & 0 & 0 & 은 & 0 & 0 & 0 & 0 & 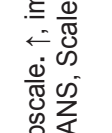 \\
\hline 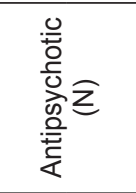 & 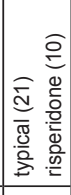 & 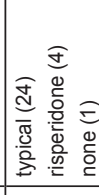 & 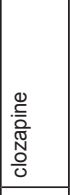 & 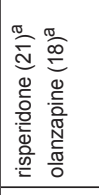 & 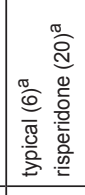 & 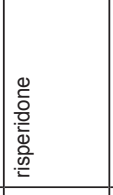 & 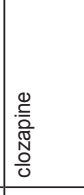 & 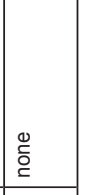 & 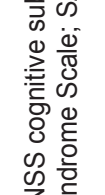 \\
\hline 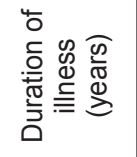 & $\begin{array}{ll}- & 0 \\
0 & 0 \\
0 & 0 \\
+1 & +1 \\
0 & 0 \\
0 & 0 \\
0 & \infty\end{array}$ & 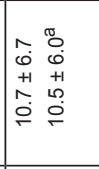 & 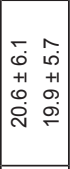 & z & 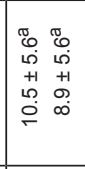 & q & $\frac{\eta}{2}$ & 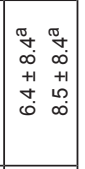 & 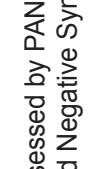 \\
\hline 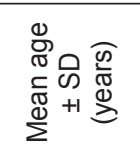 & 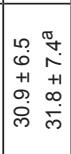 & 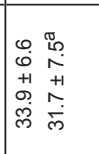 & $\mid \begin{array}{ll}0 & 0 \\
0 & 0 \\
0 & 0 \\
0 & 1 \\
0 & 0 \\
\dot{\gamma} & 0 \\
0 & 0 \\
0\end{array}$ & 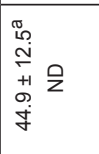 & 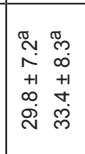 & 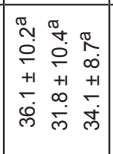 & 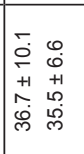 & 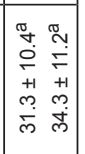 & 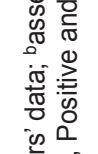 \\
\hline \multirow[t]{2}{*}{$\begin{array}{l}\frac{n}{0} \\
\frac{0}{0} \\
0 \\
\omega\end{array}$} & 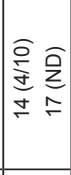 & 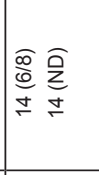 & 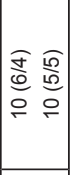 & 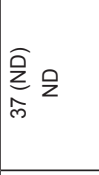 & 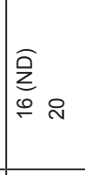 & 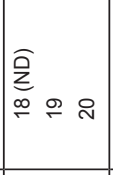 & 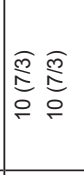 & $\sum_{0} \underline{0}$ & 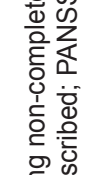 \\
\hline & $\pm \stackrel{\infty}{\simeq}$ & $\pm=$ & $\div \circ$ & gi & $\mp \bar{N}$ & $\bar{\sim} \bar{\sim} \approx$ & $\circ \circ$ & $\theta=$ & \\
\hline $\begin{array}{l}\text { 을 } \\
\text { 힘 }\end{array}$ & 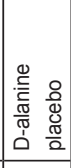 & 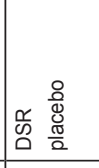 & 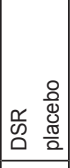 & 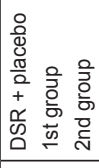 & 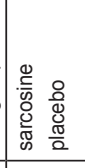 & 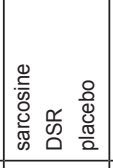 & 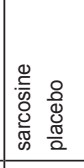 & 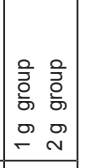 & $\frac{\bar{J}}{0}$ \\
\hline 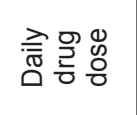 & 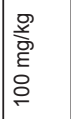 & $\begin{array}{l}\text { 胥 } \\
\text { है } \\
0 \\
\text { m }\end{array}$ & 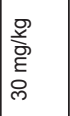 & 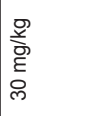 & 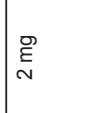 & D & I & 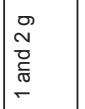 & \\
\hline 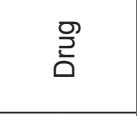 & 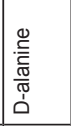 & 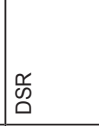 & 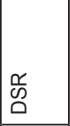 & 哭 & 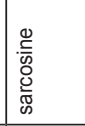 & 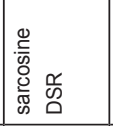 & 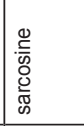 & 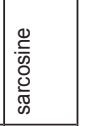 & \\
\hline $\begin{array}{l}\text {. } \\
\text { D. } \\
0 \\
0\end{array}$ & 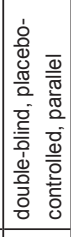 & 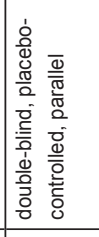 & 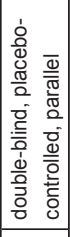 & 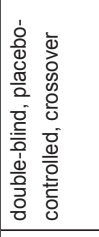 & 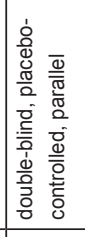 & 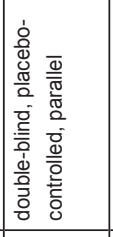 & 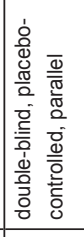 & 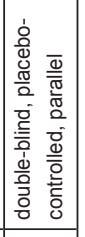 & $\begin{array}{l}\stackrel{\oplus}{\longleftarrow} \\
\stackrel{5}{\circ}\end{array}$ \\
\hline 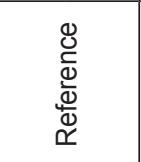 & 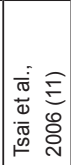 & 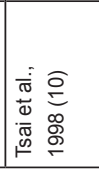 & 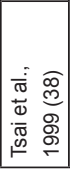 & 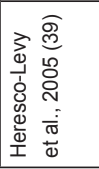 & 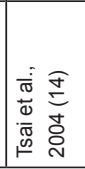 & 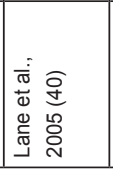 & 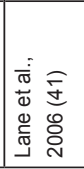 & 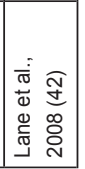 & 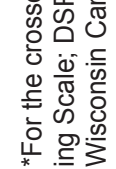 \\
\hline
\end{tabular}


tion of D-alanine $\left(100 \mathrm{mg} \cdot \mathrm{kg}^{-1} \cdot \mathrm{day}^{-1}\right)$ to typical antipsychotics and to risperidone (11). This study showed effectiveness of D-alanine adjuvant treatment to both typical antipsychotics and risperidone on the positive, negative and cognitive domains of schizophrenia. Cognitive functioning was assessed by the cognitive subscale of PANSS, with no specific cognitive tests. Furthermore, this trial had a small sample size, with larger trials being needed to confirm these initial results.

\section{D-serine}

There were four trials with the addition of D-serine to the usual antipsychotic treatment $(10,38-40)$. One trial (10) evaluated the addition of D-serine to typical antipsychotics, with amelioration of positive, negative and cognitive symptoms. Three trials $(10,39,40)$ evaluated the addition of D-serine to second-generation antipsychotics, and two of them $(10,39)$ demonstrated good outcomes regarding positive, negative and cognitive symptoms. However, one study (40) did not find beneficial results in any of the three domains. One trial with addition of D-serine to clozapine (38) showed no significant effect on the three core symptoms of schizophrenia.

\section{Sarcosine}

The first trial (14) evaluated the addition of $2 \mathrm{~g}$ sarcosine to typical antipsychotics and to risperidone, revealing an improvement of the positive, negative and cognitive symptoms of schizophrenia. The second trial (40) evaluated the addition of sarcosine to risperidone in patients with acute exacerbation of schizophrenia, observing an overall reduction of symptoms.

These results encouraged research on sarcosine as add-on therapy to clozapine (41), which led to no significant change in schizophrenia symptoms. Moreover, Lane et al. (42) evaluated the effect of sarcosine monotherapy on 12 acutely symptomatic drug-free patients with schizophrenia, who were randomly assigned to take 1 or $2 \mathrm{~g}$ sarcosine. Overall, there was no significant effect of sarcosine dose. However, the 5 responders were drug-naive patients.

\section{Minocycline}

To the best of our knowledge, the antipsychotic efficacy of minocycline was first observed in a patient with Huntington's disease (18). There is one case report (24) with addition of minocycline to the antipsychotic treatment as usual, using a dose of $150 \mathrm{mg} / \mathrm{day}$ in two cases of acute catatonic schizophrenia. Only the PANSS was used weekly for the evaluation of clinical efficacy, showing a marked reduction of positive, negative and cognitive symptoms. There were no considerable side effects, which were clinically evaluated, without using scales. The authors reported a considerable decrease of psychiatric symptoms, followed by a worsening of symptoms after discontinuation of minocycline. However, improvement was observed again after the reintroduction of minocycline. Both patients remained on minocycline co-treatment and were practically symptom-free after one year of follow-up, even though the dose of haloperidol was reduced.

Levkovitz et al. (23) examined the efficacy of minocycline as add-on treatment in a double-blind, randomized placebo-controlled study conducted on patients with early schizophrenia, who were treated with minocycline $(\mathrm{N}=$ 36 ) or placebo $(N=18)$. The patients were followed-up for 6 months with clinical evaluations by SANS and CGI. The cognitive assessment consisted of a computerized battery. This trial demonstrated improvement of negative symptoms and executive functioning. It is noteworthy that positive symptoms were not rated.

Miyaoka et al. (51) published an open-label study of minocycline as adjunctive therapy for schizophrenia. The researchers evaluated the addition of minocycline (150 mg/day) to second-generation antipsychotics in 22 patients with schizophrenia for a period of 4 weeks. The clinical ratings were performed using the PANSS, and the positive and negative symptom subscales were reduced to 40.4 and $44.0 \%$, respectively, after 4 weeks of minocycline co-treatment. The PANSS general psychopathology subscale was reduced to $52.1 \%$. All the improvements on the PANSS subscales were maintained when scored at the 4-week follow-up time.

\section{Discussion}

\section{Possible limitations of glycinergic agents}

\section{NMDA agonists}

Initial results with glycine and D-cycloserine in addition to typical and second-generation antipsychotics have demonstrated a decrease in negative and cognitive symptoms. However, the CONSIST (31) results suggested that neither glycine nor D-cycloserine was effective for the treatment of negative symptoms or cognitive impairment. Nevertheless, this trial had a low percentage of subjects treated with typical antipsychotics.

Additionally, the CONSIST (31) data showed no correlation between plasma glycine concentration and negative symptom response, despite previous findings that plasma glycine levels predicted the reduction of negative symptoms. Thus, the correlation of plasma glycine levels and negative symptom response requires further studies.

Overall, the CONSIST (31) results contested the previous hypotheses of amelioration of negative symptoms and cognitive impairment by glycine and D-cycloserine, particularly when added to second-generation antipsychotics.

There is only one trial (11) with the addition of D- 
alanine to treatment with typical antipsychotics and risperidone. This research showed effectiveness on the positive, negative and cognitive domains of schizophrenia.

The trials of D-serine added to both typical and second-generation antipsychotics $(10,39)$ indicated a broad symptomatic response in the treatment of chronic schizophrenia, but not for acute exacerbation (40).

\section{Glycine transporter-1 inhibitors}

The presence of endogenous mechanisms that regulate the impact of amino acids administered exogenously (8), in addition to the need for high oral doses and relative lack of penetration into the CNS (52), is a possible limitation of glycinergic agents. Moreover, glycine transporters regulate glycine levels in the synaptic cleft. This may limit the impact of dietary glycine in the same way that the serotonin precursor L-tryptophan is less efficient in increasing serotoninergic transmission in comparison to the selective serotonin reuptake inhibitors. As a result, glycine-T1 antagonists may be more effective in increasing synaptic glycine and effectively potentiating NMDA-R transmission (52). Several glycineT1 inhibitors are in clinical and preclinical testing, and inhibitors with greater potency are expected to be even more effective (8). In view of the early stage of development of these compounds, clinical findings are still limited to sarcosine.

Adjunctive therapy with sarcosine has provided evidence of broad amelioration of schizophrenia symptoms, but with no significant results in monotherapy (42). As a result, sarcosine has a good potential as adjunctive therapy in addition to typical antipsychotics and risperidone.

\section{Clozapine and NMDA-R}

The trials that attempted to enhance the effect of clozapine have not been effective or have worsened the psychotic frame, suggesting that glycinergic drugs interfere with the mechanism of action of clozapine. These results also indicate that clozapine has a particular efficacy related in part to the potentiation of neurotransmission mediated by NMDA receptors. In fact, clozapine has been effective against the neurotoxicity of NMDA antagonists in animal models (53). Additionally, a partial agonist of muscarinic $\mathrm{M} 1$ receptors and an agonist of dopamine D1 receptors have been shown to enhance NMDA-R function (53). Hence, clozapine is a potent partial agonist of $\mathrm{M} 1$ receptors and a partial agonist of $D 1$ receptors, which may increase NMDA-R transmission, explaining the unique efficacy of this agent (53).

\section{Minocycline}

The trials with minocycline add-on treatment to both typical and second-generation antipsychotics have shown a broad symptomatic response in schizophrenic patients.
These results warrant further investigation of minocycline add-on treatment for patients with schizophrenia.

\section{Mechanisms of action of minocycline}

The exact mechanism of action of minocycline remains elusive. In addition to inhibiting microglial activation, decreasing nitric oxide synthase and having anti-apoptotic properties, minocycline has been recently suggested to be a positive modulator of the GluR1 subunit receptors of AMPA. Although it has been previously suggested that AMPA antagonists may offer neuroprotection, Black (54) stated that some positive AMPA modulators increase brainderived neurotrophic factor production, which in turn has been reported to have neuroprotective effects. Furthermore, AMPA receptors are distinguished on the basis of subunit composition (Glu R1-4) and isoform conformation. The subunit composition of the AMPA receptor shows striking discrepancy across the brain (54) and has different properties depending on AMPA subunit or splice variant complement. Consequently, the clinical effect of an AMPA modulator may vary according to the action of different subunits.

\section{NMDA-R signaling dichotomy}

Papadia and Hardingham (55) discussed pro-survival and pro-death signaling from the NMDA-R, arguing that both excessive and poor NMDA-R activities are deleterious and cause apoptosis in neurons. For this reason, NMDA-R activity shows a bell-shaped curve of neuronal response.

Thus, the phosphoinositide-3-kinase/Akt kinase cascade is an essential pathway for the pro-survival effects of NMDA-R stimulation (55). NMDA-R activity also robustly induces cAMP-response-element-binding protein-dependent gene expression, which promotes neuronal survival (55).

On the other hand, NMDA-R activity triggers several downstream pathways of cell death by $\mathrm{Ca}^{2+}$ influx. One of these mechanisms is p38 mitogen-activated protein kinase (MAPK) activation, which can be inversely stimulated depending on NMDA-R subtype, subunit composition and localization (56).

Therefore, the magnitude of NMDA-R activation, differential synaptic versus extrasynaptic NMDA-R effects and specific subtypes of NMDA receptors can provide the key mediators of neuroprotection instead of neurodegeneration (55).

\section{Minocycline and NMDA-R}

It is important to point out that minocycline, in addition to having the ability to reverse the neurotoxic effects of NMDA antagonists, also prevented NMDA-induced injury (57).

Actually, minocycline does not have a direct action on NMDA-R and does not regulate intracellular $\mathrm{Ca}^{2+}$ influx (58). However, minocycline seems to exert a differential control of NMDA-R signaling by preserving pro-survival pathways and reducing upstream mechanisms of apoptosis. Accordingly, minocycline inhibits p38 MAPK and maintains 
the activation of phosphoinositide-3-kinase/Akt, which are prominently associated with NMDA-R neurochemistry and physiology (59).

These mechanisms may underlie the action of minocycline on caspase and cytochrome c, since they are later events in cell death. Minocycline is an anti-microglial agent and may reduce the hypothetical inflammatory component of schizophrenia. Besides, minocycline inhibits the activation of all MAPKs. It is important to emphasize that these enzymes are essential for microglia activation.

Thus, the aforementioned effects of minocycline may play an important role in the broad effect of this drug in schizophrenia as well as in a wide range of neurological disorders.

\section{Future trends and psychopharmacological targets}

The modulation of NMDA-R transmission is a promis-

\section{References}

1. Andreasen NC. Schizophrenia: the fundamental questions. Brain Res Brain Res Rev 2000; 31: 106-112.

2. Potkin SG, Jin Y, Bunney BG, Costa J, Gulasekaram B. Effect of clozapine and adjunctive high-dose glycine in treatment-resistant schizophrenia. Am J Psychiatry 1999; 156: $145-147$.

3. Hashimoto K, Engberg G, Shimizu E, Nordin C, Lindstrom $\mathrm{LH}$, lyo M. Reduced D-serine to total serine ratio in the cerebrospinal fluid of drug naive schizophrenic patients. Prog Neuropsychopharmacol Biol Psychiatry 2005; 29: 767-769.

4. Krystal JH, Karper LP, Seibyl JP, Freeman GK, Delaney $\mathrm{R}$, Bremner JD, et al. Subanesthetic effects of the noncompetitive NMDA antagonist, ketamine, in humans. Psychotomimetic, perceptual, cognitive, and neuroendocrine responses. Arch Gen Psychiatry 1994; 51: 199-214.

5. Hashimoto K, Fukushima T, Shimizu E, Komatsu N, Watanabe $\mathrm{H}$, Shinoda N, et al. Decreased serum levels of Dserine in patients with schizophrenia: evidence in support of the N-methyl-D-aspartate receptor hypofunction hypothesis of schizophrenia. Arch Gen Psychiatry 2003; 60: 572-576.

6. Heresco-Levy U, Ermilov M, Shimoni J, Shapira B, Silipo G, Javitt DC. Placebo-controlled trial of D-cycloserine added to conventional neuroleptics, olanzapine, or risperidone in schizophrenia. Am J Psychiatry 2002; 159: 480-482.

7. Heresco-Levy U, Ermilov M, Lichtenberg P, Bar G, Javitt DC. High-dose glycine added to olanzapine and risperidone for the treatment of schizophrenia. Biol Psychiatry 2004; 55: 165-171.

8. Stahl SM. Novel therapeutics for schizophrenia: targeting glycine modulation of NMDA glutamate receptors. CNS Spectr 2007; 12: 423-427.

9. Miya K, Inoue R, Takata Y, Abe M, Natsume R, Sakimura K, et al. Serine racemase is predominantly localized in neurons in mouse brain. J Comp Neurol 2008; 510: 641-654.

10. Tsai G, Yang P, Chung LC, Lange N, Coyle JT. D-serine added to antipsychotics for the treatment of schizophrenia. Biol Psychiatry 1998; 44: 1081-1089.

11. Tsai GE, Yang P, Chang YC, Chong MY. D-alanine added to antipsychotics for the treatment of schizophrenia. Biol ing therapeutic approach to the treatment of schizophrenia. However, the majority of trials had small sample sizes (less than 20 patients) and short durations. Future studies with larger numbers of subjects and longer follow-ups are needed to corroborate the initial findings.

Moreover, evaluations of potent glycine-T1 inhibitors along with other potential mechanisms for the modulation of NMDA-R neurotransmission are promising approaches to the treatment of schizophrenia. In addition, metabotropic glutamate (mGlu) receptors may be another pharmacological target for the enhancement of NMDA-R transmission. Accordingly, positive modulators of mGlu5 receptors enhance NMDA-R transmission and agonists of $\mathrm{mGlu} 2 / 3$ receptors regulate the release of glutamate (60). Another possibility lies in the combination of drugs with different mechanisms of NMDA-R potentiation.

Psychiatry 2006; 59: 230-234.

12. Goff DC, Herz L, Posever T, Shih V, Tsai G, Henderson DC, et al. A six-month, placebo-controlled trial of D-cycloserine co-administered with conventional antipsychotics in schizophrenia patients. Psychopharmacology 2005; 179: 144150.

13. Watson GB, Bolanowski MA, Baganoff MP, Deppeler CL, Lanthorn TH. D-cycloserine acts as a partial agonist at the glycine modulatory site of the NMDA receptor expressed in Xenopus oocytes. Brain Res 1990; 510: 158-160.

14. Tsai G, Lane HY, Yang P, Chong MY, Lange N. Glycine transporter I inhibitor, N-methylglycine (sarcosine), added to antipsychotics for the treatment of schizophrenia. Biol Psychiatry 2004; 55: 452-456.

15. Tuominen HJ, Tiihonen J, Wahlbeck K. Glutamatergic drugs for schizophrenia: a systematic review and meta-analysis. Schizophr Res 2005; 72: 225-234.

16. Shim SS, Hammonds MD, Kee BS. Potentiation of the NMDA receptor in the treatment of schizophrenia: focused on the glycine site. Eur Arch Psychiatry Clin Neurosci 2008; 258: 16-27.

17. Domercq M, Matute C. Neuroprotection by tetracyclines. Trends Pharmacol Sci 2004; 25: 609-612.

18. Denovan-Wright EM, Devarajan S, Dursun SM, Robertson HA. Maintained improvement with minocycline of a patient with advanced Huntington's disease. J Psychopharmacol 2002; 16: 393-394.

19. Zhang L, Shirayama $Y$, Shimizu E, lyo M, Hashimoto $K$. Protective effects of minocycline on 3,4-methylenedioxymethamphetamine-induced neurotoxicity in serotonergic and dopaminergic neurons of mouse brain. Eur $J$ Pharmacol 2006; 544: 1-9.

20. Zhang L, Shirayama Y, lyo M, Hashimoto K. Minocycline attenuates hyperlocomotion and prepulse inhibition deficits in mice after administration of the NMDA receptor antagonist dizocilpine. Neuropsychopharmacology 2007; 32: 20042010.

21. Fujita $Y$, Ishima $T$, Kunitachi $S$, Hagiwara $H$, Zhang L, Iyo $M$, et al. Phencyclidine-induced cognitive deficits in mice are 
improved by subsequent subchronic administration of the antibiotic drug minocycline. Prog Neuropsychopharmacol Biol Psychiatry 2008; 32: 336-339.

22. Levkovitz $Y$, Levi U, Braw Y, Cohen H. Minocycline, a second-generation tetracycline, as a neuroprotective agent in an animal model of schizophrenia. Brain Res 2007; 1154: 154-162.

23. Levkovitz $\mathrm{Y}$, Mendlovic S, Issak Si, Braw $\mathrm{Y}$, LevkovitchVerbin $\mathrm{H}$, Gal G, et al. Minocycline ameliorates negative symptoms and cognitive deficits in early-phase schizophrenia: a double-blind, randomized placebo-controlled study. Schizophr Res 2008; 14 (Abstract).

24. Miyaoka T, Yasukawa R, Yasuda H, Hayashida M, Inagaki $\mathrm{T}$, Horiguchi J. Possible antipsychotic effects of minocycline in patients with schizophrenia. Prog Neuropsychopharmacol Biol Psychiatry 2007; 31: 304-307.

25. Javitt DC, Zylberman I, Zukin SR, Heresco-Levy U, Lindenmayer JP. Amelioration of negative symptoms in schizophrenia by glycine. Am J Psychiatry 1994; 151: 1234-1236.

26. Heresco-Levy U, Javitt DC, Ermilov M, Mordel C, Horowitz A, Kelly D. Double-blind, placebo-controlled, crossover trial of glycine adjuvant therapy for treatment-resistant schizophrenia. Br J Psychiatry 1996; 169: 610-617.

27. Heresco-Levy U, Javitt DC, Ermilov M, Mordel C, Silipo G, Lichtenstein M. Efficacy of high-dose glycine in the treatment of enduring negative symptoms of schizophrenia. Arch Gen Psychiatry 1999; 56: 29-36.

28. Evins AE, Fitzgerald SM, Wine L, Rosselli R, Goff DC. Placebo-controlled trial of glycine added to clozapine in schizophrenia. Am J Psychiatry 2000; 157: 826-828.

29. Javitt DC, Silipo G, Cienfuegos A, Shelley AM, Bark N, Park $M$, et al. Adjunctive high-dose glycine in the treatment of schizophrenia. Int J Neuropsychopharmacol 2001; 4: 385391.

30. Diaz P, Bhaskara S, Dursun SM, Deakin B. Double-blind, placebo-controlled, crossover trial of clozapine plus glycine in refractory schizophrenia negative results. J Clin Psychopharmacol 2005; 25: 277-278.

31. Buchanan RW, Javitt DC, Marder SR, Schooler NR, Gold JM, McMahon RP, et al. The Cognitive and Negative Symptoms in Schizophrenia Trial (CONSIST): the efficacy of glutamatergic agents for negative symptoms and cognitive impairments. Am J Psychiatry 2007; 164: 1593-1602.

32. Rosse RB, Fay-McCarthy M, Kendrick K, Davis RE, Deutsch SI. D-cycloserine adjuvant therapy to molindone in the treatment of schizophrenia. Clin Neuropharmacol 1996; 19: 444-450.

33. Heresco-Levy U, Javitt DC, Ermilov M, Silipo G, Shimoni J. Double-blind, placebo-controlled, crossover trial of Dcycloserine adjuvant therapy for treatment-resistant schizophrenia. Int J Neuropsychopharmacol 1998; 1: 131-135.

34. Goff DC, Tsai G, Levitt J, Amico E, Manoach D, Schoenfeld $\mathrm{DA}$, et al. A placebo-controlled trial of D-cycloserine added to conventional neuroleptics in patients with schizophrenia. Arch Gen Psychiatry 1999; 56: 21-27.

35. Goff DC, Henderson DC, Evins AE, Amico E. A placebocontrolled crossover trial of D-cycloserine added to clozapine in patients with schizophrenia. Biol Psychiatry 1999; 45: 512514.

36. van Berckel BN, Evenblij CN, van Loon BJ, Maas MF, van der Geld MA, Wynne HJ, et al. D-cycloserine increases positive symptoms in chronic schizophrenic patients when administered in addition to antipsychotics: a double-blind, parallel, placebo-controlled study. Neuropsychopharmacology 1999; 21: 203-210.

37. Duncan EJ, Szilagyi S, Schwartz MP, Bugarski-Kirola D, Kunzova A, Negi S, et al. Effects of D-cycloserine on negative symptoms in schizophrenia. Schizophr Res 2004; 71: 239-248.

38. Tsai GE, Yang P, Chung LC, Tsai IC, Tsai CW, Coyle JT. Dserine added to clozapine for the treatment of schizophrenia. Am J Psychiatry 1999; 156: 1822-1825.

39. Heresco-Levy U, Javitt DC, Ebstein R, Vass A, Lichtenberg $P$, Bar $G$, et al. D-serine efficacy as add-on pharmacotherapy to risperidone and olanzapine for treatment-refractory schizophrenia. Biol Psychiatry 2005; 57: 577-585.

40. Lane HY, Chang YC, Liu YC, Chiu CC, Tsai GE. Sarcosine or $D$-serine add-on treatment for acute exacerbation of schizophrenia: a randomized, double-blind, placebo-controlled study. Arch Gen Psychiatry 2005; 62: 1196-1204.

41. Lane HY, Huang CL, Wu PL, Liu YC, Chang YC, Lin PY, et al. Glycine transporter I inhibitor, N-methylglycine (sarcosine), added to clozapine for the treatment of schizophrenia. Biol Psychiatry 2006; 60: 645-649.

42. Lane HY, Liu YC, Huang CL, Chang YC, Liau CH, Perng $\mathrm{CH}$, et al. Sarcosine (N-methylglycine) treatment for acute schizophrenia: a randomized, double-blind study. Biol Psychiatry 2008; 63: 9-12.

43. Rosse RB, Theut SK, Banay-Schwartz M, Leighton M, Scarcella E, Cohen CG, et al. Glycine adjuvant therapy to conventional neuroleptic treatment in schizophrenia: an open-label, pilot study. Clin Neuropharmacol 1989; 12: 416424.

44. Costa J, Khaled E, Sramek J, Bunney W Jr, Potkin SG. An open trial of glycine as an adjunct to neuroleptics in chronic treatment-refractory schizophrenics. J Clin Psychopharmacol 1990; 10: 71-72.

45. Leiderman E, Zylberman I, Zukin SR, Cooper TB, Javitt DC. Preliminary investigation of high-dose oral glycine on serum levels and negative symptoms in schizophrenia: an openlabel trial. Biol Psychiatry 1996; 39: 213-215.

46. Cascella NG, Macciardi F, Cavallini C, Smeraldi E. Dcycloserine adjuvant therapy to conventional neuroleptic treatment in schizophrenia: an open-label study. J Neural Transm Gen Sect 1994; 95: 105-111.

47. Goff DC, Tsai G, Manoach DS, Coyle JT. Dose-finding trial of $\mathrm{D}$-cycloserine added to neuroleptics for negative symptoms in schizophrenia. Am J Psychiatry 1995; 152: 1213-1215.

48. Goff DC, Tsai G, Manoach DS, Flood J, Darby DG, Coyle JT. D-cycloserine added to clozapine for patients with schizophrenia. Am J Psychiatry 1996; 153: 1628-1630.

49. van Berckel BN, Hijman R, van der Linden JA, Westenberg HG, van Ree JM, Kahn RS. Efficacy and tolerance of D-cycloserine in drug-free schizophrenic patients. Biol Psychiatry 1996; 40: 1298-1300.

50. Evins AE, Amico E, Posever TA, Toker R, Goff DC. Dcycloserine added to risperidone in patients with primary negative symptoms of schizophrenia. Schizophr Res 2002; 56: 19-23.

51. Miyaoka T, Yasukawa R, Yasuda H, Hayashida M, Inagaki T, Horiguchi J. Minocycline as adjunctive therapy for schizophrenia: an open-label study. Clin Neuropharmacol 2008; 31: $287-292$.

52. Javitt DC. Glycine transport inhibitors and the treatment of 
schizophrenia. Biol Psychiatry 2008; 63: 6-8.

53. Stone JM, Morrison PD, Pilowsky LS. Glutamate and dopamine dysregulation in schizophrenia - a synthesis and selective review. J Psychopharmacol 2007; 21: 440-452.

54. Black MD. Therapeutic potential of positive AMPA modulators and their relationship to AMPA receptor subunits. A review of preclinical data. Psychopharmacology 2005; 179: 154-163.

55. Papadia S, Hardingham GE. The dichotomy of NMDA receptor signaling. Neuroscientist 2007; 13: 572-579.

56. Waxman EA, Lynch DR. N-methyl-D-aspartate receptor subtype mediated bidirectional control of p38 mitogen-activated protein kinase. J Biol Chem 2005; 280: 29322-29333.

57. Song $Y$, Wei EQ, Zhang WP, Ge QF, Liu JR, Wang ML, et al. Minocycline protects PC12 cells against NMDA-induced injury via inhibiting 5-lipoxygenase activation. Brain Res 2006; 1085: 57-67.

58. Pi R, Li W, Lee NT, Chan HH, Pu Y, Chan LN, et al. Minocycline prevents glutamate-induced apoptosis of cerebellar granule neurons by differential regulation of p38 and Akt pathways. J Neurochem 2004; 91: 1219-1230.

59. Haddad JJ. N-methyl-D-aspartate (NMDA) and the regulation of mitogen-activated protein kinase (MAPK) signaling pathways: a revolving neurochemical axis for therapeutic intervention? Prog Neurobiol 2005; 77: 252-282.

60. Moghaddam B. Bringing order to the glutamate chaos in schizophrenia. Neuron 2003; 40: 881-884. 